\title{
Confined modes in photonic microtube structures
}

\author{
John F. Donegan*ab, Yury P. Rakovich ${ }^{\mathrm{a}}$, Yurii Gun'ko ${ }^{\mathrm{c}}$, Tania S. Perova ${ }^{\mathrm{d}}$ and R. Alan Moore ${ }^{\mathrm{d}}$ \\ ${ }^{a}$ Semiconductor Photonics Group, School of Physics, Trinity College, Dublin 2, Ireland. \\ ${ }^{\mathrm{b}}$ Principal Investigator, CRANN Research Centre, Trinity College Dublin \\ ${ }^{\mathrm{c} S}$ School of Chemistry, Trinity College, Dublin 2, Ireland \\ ${ }^{\mathrm{d}}$ School of Engineering, Trinity College, Dublin 2, Ireland
}

\begin{abstract}
We present the results of studies on small photonic microtube structures of less than 10 microns diameter. A new technique based on vacuum assisted filtration is used to produce the microtube resonantors. Whispering gallery modes are probed through the photoluminescence emission from the glass material forming the cavity. We observe resonances with polarized emission having quality factors upto 3000 . These microresonator structures exhibit a large evanescent field which makes them interesting for potential photonic applications.
\end{abstract}

Keywords: Microspheres, nanocrystals, microtube, resonators, photoluminescence, whispering gallery modes

\section{INTRODUCTION}

Optical microcavities, which confine the propagation of light in all three dimensions, are extensively studied due to their microscopic size (1-500 microns), high quality factor Q $\left(10^{2}-10^{9}\right)$ and the possibility of achieving low threshold lasing (less than $1 \mu \mathrm{W}) .{ }^{1-8}$ These systems exhibit novel features arising from our ability to tailor the electro-magnetic field and the photon density of states in a prescribed manner, such as to concentrate and guide light on the micrometer scale. For structures of less than 100 microns and particularly for those less than 10 microns, the quality factors are $10^{4}$ or less and the modes are spectrally well spaced. However, it is the evanescent field of the cavity modes that extend many microns outside the cavity that make such structures interesting, in particular opening up the possibility of coupling microcavities to produce new photonic structures.

Among the microcavity systems which have been developed during the past 10-15 years, optical emitters with cylindrical symmetry which support whispering gallery modes (WGMs) have attracted particular interest. ${ }^{2-4}$ The resonantly enhanced optical response make these high-Q microcavities attractive as novel building blocks for photonic devices. The cylindrical cavity format is also compatible with a large variety of sensing modalities such as immunoassay and molecular diagnostic assay. ${ }^{9,10}$

Experimentally, the most widely studied configuration of thin-wall microtube cavities is the microcapillary filled with a highly-luminescent dye solution. ${ }^{3,4}$ The diameter (typically 50-200 $\mu \mathrm{m}$ ) and wall thickness of these microcapillaries can be controlled by the etching commercially-available glass samples in an HF-water solution. In the small-size regime (diameter $<10 \mu \mathrm{m}$ ), semiconductor microdisks or micropillars of finite height have been widely used as tools to control spontaneous emission and confine photons in three dimensions. ${ }^{2}$

The synthesis of tubular materials such as silica, magnesium oxide, titanium dioxide and zinc oxide has been reported. ${ }^{11}$ Along with these techniques the fabrication of micro-wire arrays in situ using electrochemical ${ }^{12-14}$ and electroless ${ }^{15-17}$ deposition in a porous template has proven to be a superior option for preparing more complex, higher aspect ratio 3-D microtube and microwire arrays. ${ }^{18}$ The formation of luminescent structures by incorporating rare-earth ions into a porous matrix using various techniques like ion-implantation, ${ }^{19}$ electrochemical migration, ${ }^{20}$ or the spin-on technique ${ }^{21}$ has been demonstrated. However, the fabrication of small $(<10 \mu \mathrm{m}$ diameter $)$ high-Q cylindrical microcavities generally involves complex and time-consuming processing., ${ }^{2,22}$ Here we describe a new and very straightforward method for fabricating highly-luminescent small aluminosilicate microtubes of $\sim 6-7 \mu \mathrm{m}$ outer diameter, which are shown to act as efficient optical cylindrical micro-resonators.

*jdonegan@tcd.ie; phone +353-1-8961987; fax +353-1-6711759; www.tcd.ie/Physics/Optoelectronics

Laser Resonators and Beam Control IX, edited by Alexis V. Kudryashov, Alan H. Paxton, Vladimir S. Ilchenko, Proc. of SPIE Vol. 6452, 64520Y, (2007) · 0277-786X/07/\$15 - doi: 10.1117/12.714362 


\section{EXPERIMENTAL}

In our fabrication approach, we took advantage of the well-developed sol-gel technique combined with the versatility of ordered porous membranes as templates. $\mathrm{Si}\left(\mathrm{OC}_{2} \mathrm{H}_{5}\right)_{4}$ was first hydrolysed for $1 \mathrm{~h}$ at room temperature with a solution of $\mathrm{H}_{2} \mathrm{O}, \mathrm{C}_{2} \mathrm{H}_{5} \mathrm{OH}$ and $\mathrm{HCl}$ in the molar ratios 1: 1: 0.0027 per mole of $\mathrm{Si}\left(\mathrm{OC}_{2} \mathrm{H}_{5}\right)_{4}$ respectively. Then $\mathrm{Al}\left(\mathrm{OC}_{4} \mathrm{H}_{9}{ }^{\mathrm{sec}}\right)_{3}$ was added to this solution and the mixture was stirred for $15 \mathrm{~min}$ at $70^{\circ} \mathrm{C}$. The resultant homogenous mixture was hydrolysed by adding the mixed solution of $\mathrm{H}_{2} \mathrm{O}, \mathrm{C}_{2} \mathrm{H}_{5} \mathrm{OH}$ and $\mathrm{HCl}$ in the molar ratio 4: 1: 0.011 per mole of alkoxide, respectively. Finally, 30 minutes stirring provided the conversion of this sol to $5 \mathrm{Al}_{2} \mathrm{O}_{3} .95 \mathrm{SiO}_{2}(\mathrm{~mol} \%)$ alumosilicate gel. The sol, just before its gelation point was then placed on the top of the micro-channel glass samples and 30 mbar vacuum-assisted filtration resulted in the formation of microtubes inside the channels. Following drying at room temperature for 1 day and further annealing at $500^{\circ} \mathrm{C}$ for 2 hours, the fabricated microtubes were isolated by mechanical destruction of the template.

The steady-state PL spectra from both a single microtube and microtubes within the matrix were recorded in a backscattering geometry using a RENISHAW micro-Raman system $\left(1800 \mathrm{~mm}^{-1}\right.$ grating, $>1 \mathrm{~cm}^{-1}$ resolution) equipped with a microscope objective $(\times 100)$, a notch and plasma filters and a CCD camera. An $\mathrm{Ar}^{+}$laser (wavelength $514.5 \mathrm{~nm}$ ) was used as the excitation source. PL decays were measured using time-correlated single-photon counting (Time-Harp, PicoQuant). The samples were excited by $480 \mathrm{~nm}$ picosecond pulses generated by a PicoQuant, LDH-480 laser head contolled by a PDL-800B driver. The setup was operated at a $20 \mathrm{-MHz}$ repetition rate with an overall time resolution of $150 \mathrm{psec}$. Fluorescence lifetime images from the microtube were collected with the FLIM system (Microtime200 timeresolved confocal microscope system, PicoQuant) equipped with an Olympus IX71 inverted microscope. Lifetime maps were calculated on a pixel-by-pixel basis by fitting the lifetime of each pixel to the logarithm of the intensity. The FLIM system response was negligible compared with typical lifetimes of the aluminosilicate microtubes $(\sim 3-10 \mathrm{~ns})$.

The scanning electron microscopy (SEM), images of the samples were obtained using an FESEM (Hitachi S-4300 scanning electron microscope), which was operated at $5.0 \mathrm{kV}$ or $20 \mathrm{kV}$. The beam-specimen interaction produces a variety of signals including secondary electrons (SE), back-scattered electrons (BE) and X-rays. The low energy secondary electrons are collected to form the standard image and the high energy back-scattered electrons provide an image with good atomic number contrast. Samples for analysis were mounted on aluminium stubs and coated with gold.

\section{RESULTS AND DISCUSSION}

The fabrication of luminescent aluminosilicate microtubes was achieved by vacuum-assisted infiltration of freshly prepared alumosilicate gel through a micro-channel glass template.

Cross-sectional Scanning Electron Microscopy (SEM) images of the microtubes are presented in Figure 1. From these images it can be seen that the microtubes are hollow. The images also show the uniform distribution of the microtubes inside the porous matrix. From the SEM pictures, we find that the microtubes have an outer diameter of 6-7 $\mu \mathrm{m}$ and an inner diameter of $2.7-3 \mu \mathrm{m}$. The maximum length of a single microtube is $200 \mu \mathrm{m}$. Thus, the SEM images show the formation of high aspect ratio microtubes having smooth walled structures, ideal for photonic studies.

The formation of alumosilicate microtubes by vacuum filtration of the gel can be explained by the fact that the cohesive driving forces for complete microchannel filling are substantially weaker than the adhesive forces to the pore walls. Similar phenomena have been reported for the formation of polymer nanotubes by wetting of ordered porous templates using a polymer melt. ${ }^{23}$

The thermal treatment of the samples resulted in highly emissive air-stable structures which display broad-band visible photoluminescence (PL) originating from carbon defects substituting for silicon in the microtube material. In this case the thermal decomposition of organic (e.g. alkoxide) groups in the xerogel results in a substitution of $\mathrm{Si}$ atoms with carbon giving strongly luminescent species. A similar type of observation for silicates was reported before by M. J. Sailor et al.$^{24}$ An elemental analysis of the annealed aluminosilicate gel showed carbon and hydrogen content of $0.32 \%$ and of $0.56 \%$ respectively. The PL spectrum of a single aluminosilicate microtube accommodated in the microporous glass substrate is presented in Figure 2a. 

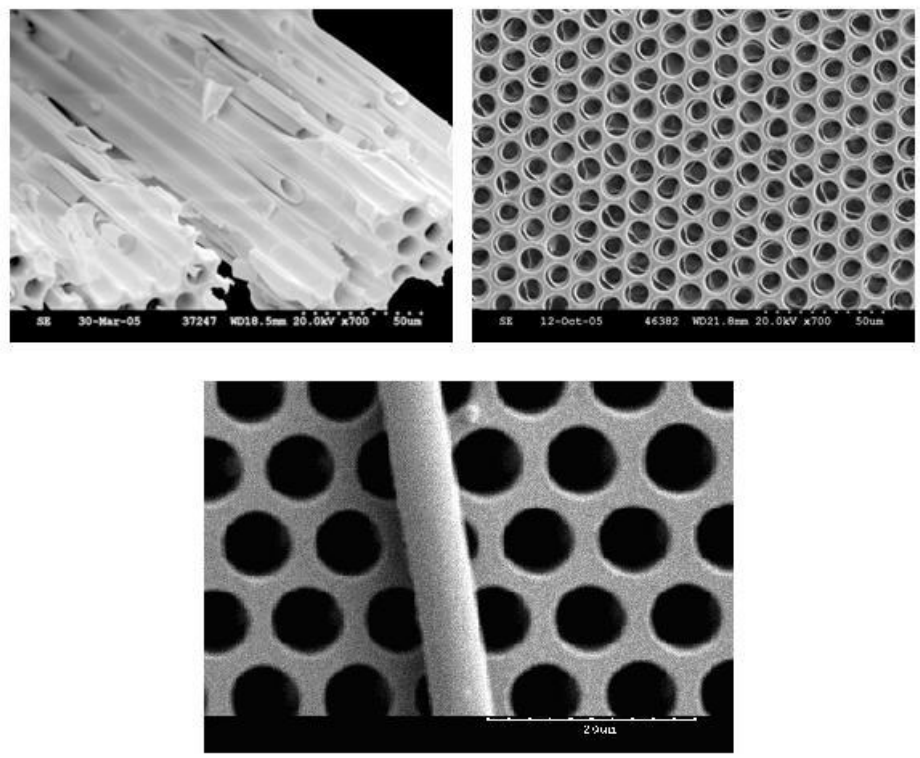

Figure 1. Cross-sectional and plan view SEM images of the microtubes inside and outside the matrix.

The emission spectrum shows a broad luminescence band ranging from 520 to $800 \mathrm{~nm}$. The PL of a single aluminosilicate microtube shows a clear multi-exponential decay. The observed multi-exponential decay kinetics are consistent with a PL model that includes multiple emission pathways and is indicative of a broad lifetime distribution caused by the corresponding distribution of defect or trap states due to the amorphous nature of the microtube material (Figure 2b).
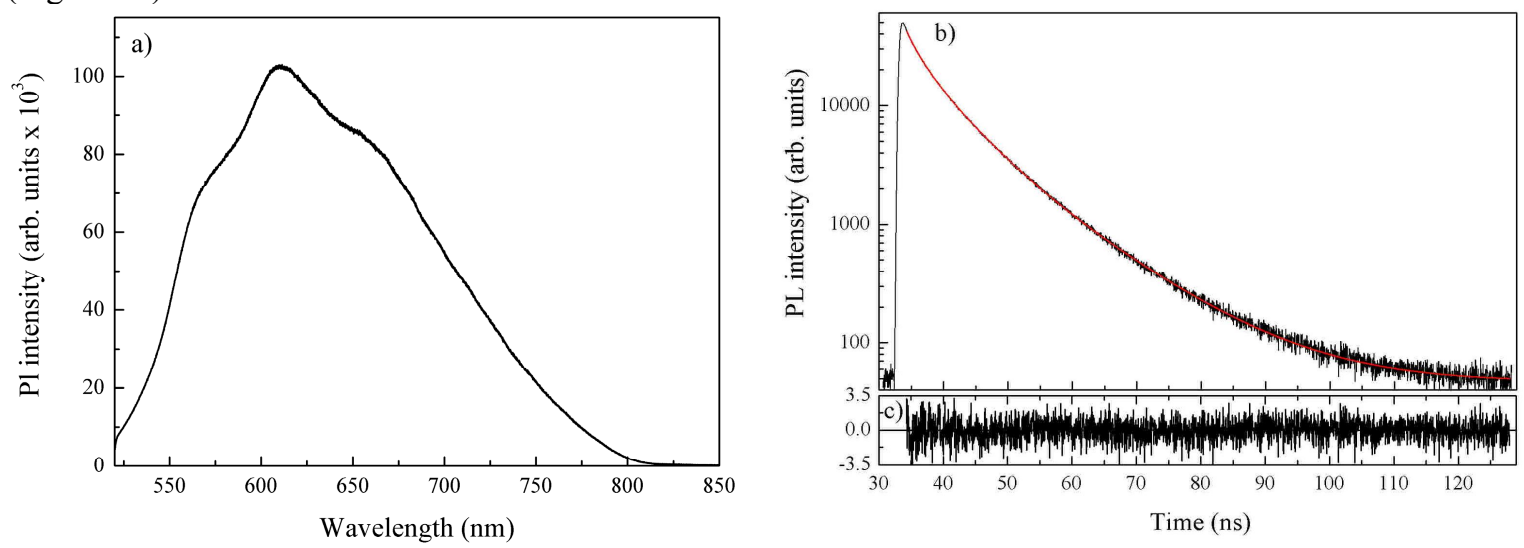

Figure 2.(a) PL spectra and (b) time-dependent PL intensity decay (three exponential fit) of a single aluminosilicate microtube accommodated in a glass microchannel matrix. (c) residuals of the fitting process.

In the case when fluorophores are embedded in nonuniform environments, it has been shown that PL decays can be best understood by a model of continuous distributions of decay times. ${ }^{25}$ In this case fitting procedures cannot distinguish sufficiently between, for example, a single Gaussian distribution of lifetimes and the sum of two exponentials or a bimodal Gaussian distribution and the sum of at least three exponentials. ${ }^{26,27}$ Thus, a description based on discrete lifetime components should only be regarded as truly representing discrete molecular states if supported by supplementary data. ${ }^{28}$ Therefore, to gain a better insight into the spatial distribution of lifetimes, the PL dynamics were evaluated from the FLIM (fluorescence lifetime imaging microscope) images i.e. maps of two-dimensional in-plane variations of the PL decay times.

Each pixel in the FLIM image gives the lifetime at a particular position in space $(x, y)$ while monitoring the entire PL spectrum. The lifetime image of the array of aluminosilicate microtubes in a micro-porous glass matrix (Figure $3 a$ and $b$ ) 
clearly demonstrates the distribution of emitting species over the microtube cross-section, showing that the dominant emission is at the rim of the microtube. In both images, the histograms show lifetime distributions that consist of two maxima centered at $0.7 \mathrm{~ns}$ and $3.5 \mathrm{~ns}$ with the values of full width at half maximum FWHM $=0.5 \mathrm{~ns}$ and $2 \mathrm{~ns}$ respectively. Comparing the lifetime histogram obtained from the array (Figure 3c) with that measured from a single microtube, accommodated in the porous matrix, (Figure 3d) it is very clear that there is no deviation in lifetime within the ensemble of microtubes formed in the matrix. This demonstrates the high quality of the samples and the reproducibility of the optical parameters.

When separated from the matrix, a drastic modification in lifetime distribution has been observed in the FLIM image of the individual microtube cavity (Figure 4). Once again two maxima in the lifetime histogram of the "free standing" single microtube are present. However, in contrast to the embedded microtube, the long-lived component centered at 10 ns is now dominant and is accompanied by a second smaller peak at $4 \mathrm{~ns}$. The faster decay observed in the case of the embedded microtube implies that lifetimes of the emitting states are strongly reduced, thereby demonstrating the presence of highly-efficient nonradiative energy transfer from the microtube into the surrounding matrix. In contrast, when the microtube is separated from the matrix, the nonradiative pathways introduced by the martix are no longer competing with the radiative pathways, resulting in strong enhancement of lifetimes.

It is also important to note that lifetime distribution detected from the side surface of an individual microtube (Figure 4c) is almost identical to that measured from the microtube tip (Figure 4d).
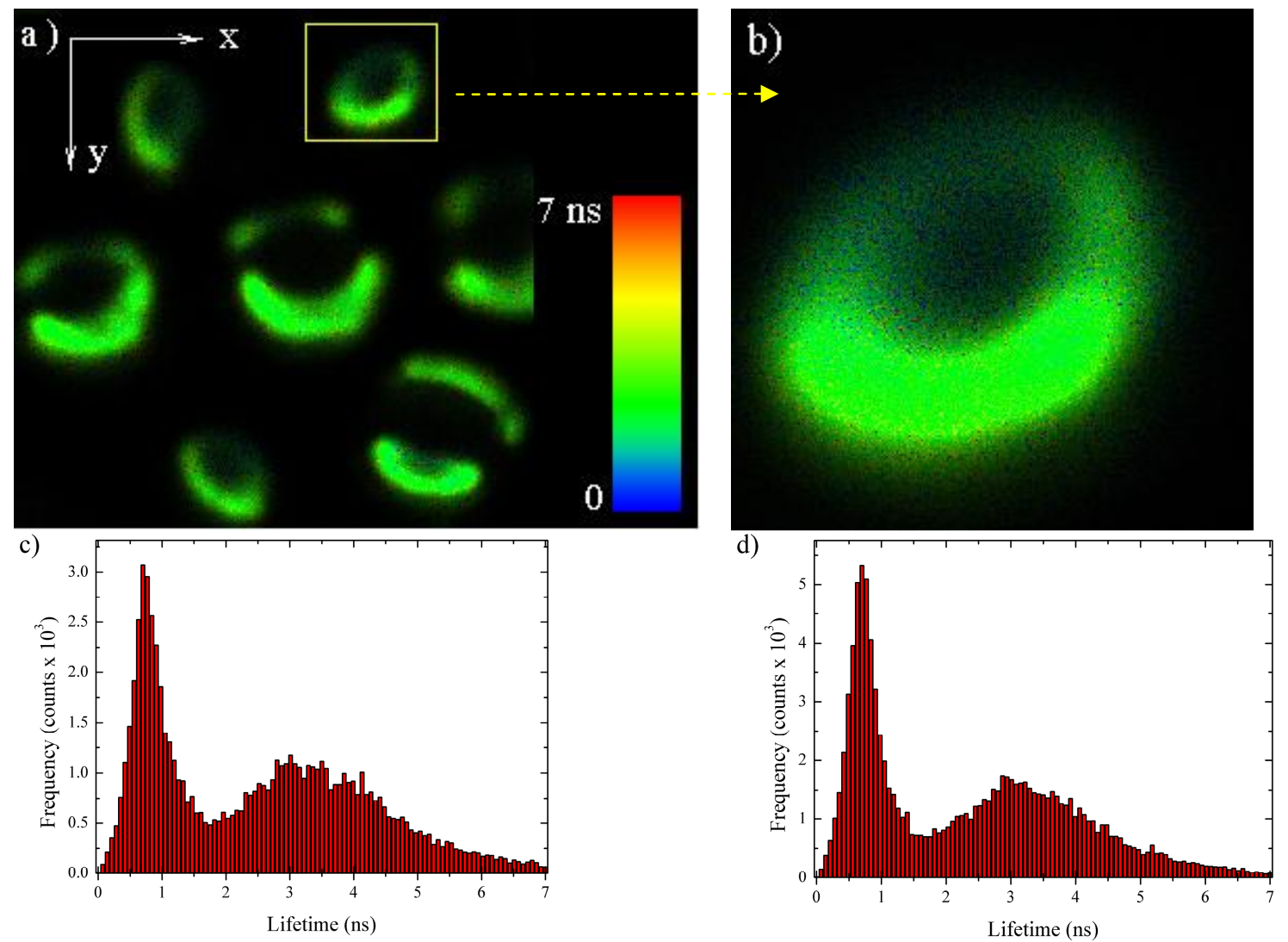

Figure 3. Fluorescence lifetime image of an array of aluminosilicate microtubes in a microporous glass matrix (a) The image was collected at $250 \times 250$ pixel resolution with 4096 time channels; 2 ms acquisition time per pixel and total recording time 6.63 min. Image size: $29.90 \mu \mathrm{m}$ x $29.90 \mu \mathrm{m}$. Panel (b) shows an enlarged image of one single aluminosilicate microtube in a glass matrix (indicated by frame in panel (a)). In this case the image was collected at the same pixel resolution and recording time, with image size $7.30 \mu \mathrm{m} \times 7.30 \mu \mathrm{m}$. Panels (c) and (d) demonstrate the corresponding lifetime histograms.

The two-peak structure shown in Figures 3 and 4 implies that at least two different mechanisms are involved in the decay processes. The peak around $3 \mathrm{~ns}$ is almost unaltered in going from the matrix to the free microtube. It is therefore part of 
the bulk of the microtube material and is unaffected by the microcavity. The defect is likely to have a deep state with a highly localized wavefunction.

On the other hand, there is a significant alteration to the short lifetime component. Its average value increases by an order of magnitude. As we will see later, the microtube microcavity structure has a large evanescent field. While in the matrix there is very strong coupling of this field to the matrix and this is removed once the microtube is free from the matrix. Very efficient transfer in a Förster energy transfer mechanism will result in nonradiative decay processes. ${ }^{29}$ This type of energy transfer can occur through dipole-dipole interactions between an emitter in the glass and the matrix surface and is strongly dependent on distance between them. The large evanescent field of the microtube will enhance the transfer rate thereby producing the short component in the lifetime of the PL within the matrix.

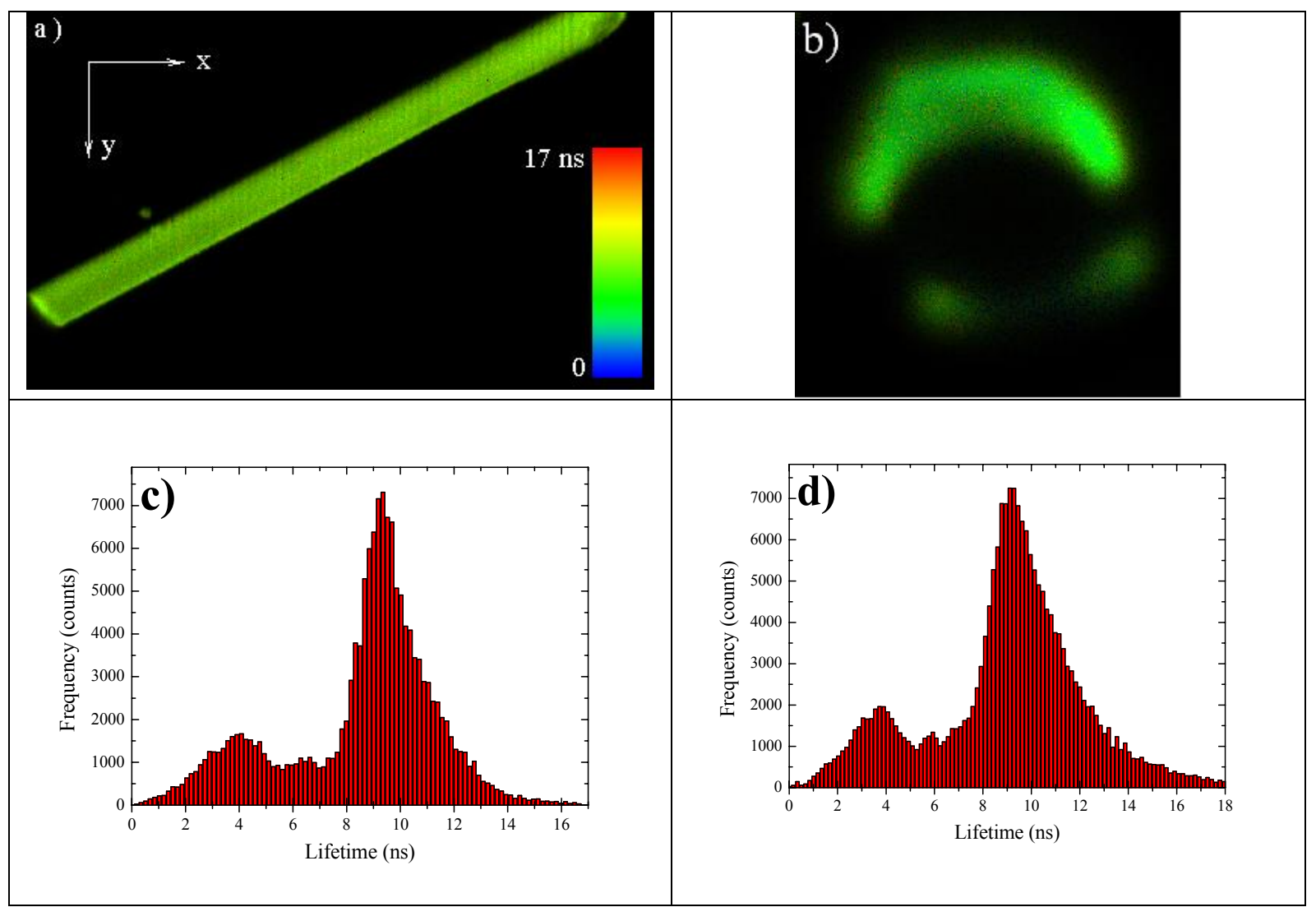

Figure 4. a) Fluorescence lifetime image of single aluminosilicate microtube. The image was collected at $416 \times 250$ pixel resolution with 4096 time channels; $2 \mathrm{~ms}$ acquisition time was provided per pixel and total recording time was $9.6 \mathrm{~min}$. Image size: $67.72 \mu \mathrm{m} \times 40.70 \mu \mathrm{m}$. b) Tip view of single the aluminosilicate microtube. Image size $7.20 \mu \mathrm{m} \times 7.20 \mu \mathrm{m}$. Panels (c) and (d) demonstrate the corresponding lifetime histograms.

In contrast to the broad PL band in the spectra of tubes embedded in a micro-porous glass matrix (Figure 2a), the emission spectra of a single "free standing" microtube exhibit very sharp periodic structure (Figure 5). When separated from the matrix, the microtube is much more optically dense than its surrounding medium allowing light propagating inside the microtube to be spatially constrained to travel along the rim of a cross-section of the microtube, and therefore it is said to be trapped in a WGM. The presence of sharp emission peaks in the spectrum of a single microtube is an immediate result of this optical confinement (Figure 5). These peaks correspond to optical resonance locations and reflect the fact that transition probabilities are increased for emission wavelengths near resonance. The WGM peaks are superimposed on a background signal arising from a part of the emission, which does not match any WGM of the microtube (Figure 5). 
In the small size regime for microcavities $(<10 \mu \mathrm{m})$ where polarized modes are well separated, the number of modes is small and they can be identified. For studies of lasing and quantum information processing in particular, there is a need to determine the mode number and polarization of the WGM resonances. In order to identify the peaks in the observed WGM structure, we have adopted the boundary-value solution to the problem of scattering of plane electromagnetic waves by a dielectric micro-cylinder. ${ }^{30,31}$ The mode identification takes account of the cylindrical symmetry of the microtubes. The extinction efficiency $\mathrm{Q}$ is derivable from Lorenz-Mie theory when the microcylinder is illuminated by monochromatic, plane polarized light with the electric vector vibrating parallel to the cylinder axis (TM polarization):

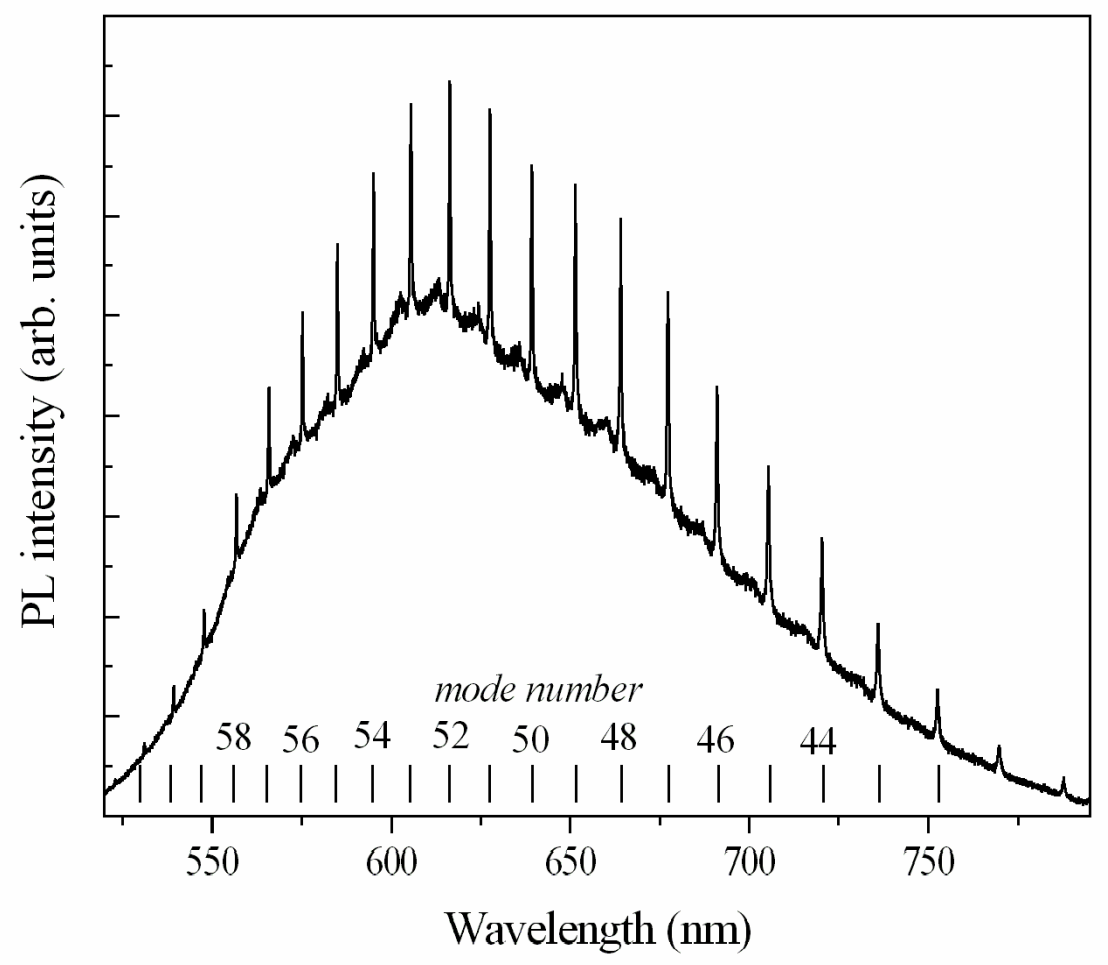

Figure 5. Room-temperature PL spectra of a single free-standing microtube. The inset shows a microscope image of the microtube, with the cross-hairs indicating the excitation-detection position.

$Q_{\text {ext }}^{T M}=\frac{2}{x} \operatorname{Re}\left[b_{0}+2 \sum_{n=1}^{\infty} b_{n}\right]$,

or for the case when the electric field is linearly polarized normal to the axis of the cylinder (TE polarization):

$Q_{e x t}^{T E}=\frac{2}{x} \operatorname{Re}\left[a_{0}+2 \sum_{n=1}^{\infty} a_{n}\right]$,

where $x=2 r / \lambda$ is the size parameter, $r$ is the radius of microcylinder, $n$ is the angular mode number. Apart from $n$ (which is proportional to the circumference of cross-section divided by the wavelength of the light propagating within the microcylinder), the spectral distributions of TM and TE WGM are characterized by the mode order $\ell$ (which indicates the number of maxima in the radial distribution of the internal electric field), and the azimuthal mode number $m$ (which gives the orientation of the WGMs orbital plane). ${ }^{32}$

In the absence of gain, the Mie scattering partial wave amplitudes $a_{n}(x, m)$ and $b_{n}(x, m)$ can be expressed in the form 
$a_{n}=\frac{J_{n}^{\prime}(m x) J_{n}(x)-m J_{n}(m x) J_{n}^{\prime}(x)}{J_{n}^{\prime}(m x) H_{n}^{(1)}(x)-m J_{n}(m x) H_{n}^{\prime(1)}(x)}$

for TE modes and

$b_{n}=\frac{m J_{n}^{\prime}(m x) J_{n}(x)-J_{n}(m x) J_{n}^{\prime}(x)}{m J_{n}^{\prime}(m x) H_{n}^{(1)}(x)-J_{n}(m x) H_{n}^{\prime(1)}(x)}$

for TM modes, where $J_{n}(x)$ is a Bessell function of the first kind, $H_{n}(x)$ is a Henkel function of the second kind, and the primes denote differentiation of the functions with respect to their arguments. According to Eqs. (1)-(2) resonance structure in scattering spectra can be expected as the real part of $a_{n}$ or $b_{n}$ reaches its maximum value of 1 and the imaginary part is passing through 0 from the positive to the negative side. In other words, the resonances in the Mie scattering characteristics occur when denominators in Eq. (3) (TE modes) or Eq (4) (TM modes) are equal to zero for a particular mode $n$.

These conditions are transcendental equations, which can be solved for the size parameter $x$ (position of resonance) for given values of refractive index and for given mode number $n$. Thus, comparing the calculated results with the spectral positions and polarization of the WGM in the experimental PL spectra, we can identify the indexes $n$ for each mode using the diameter of the microtube that was found from the SEM micrographs. The algorithm of the mode assignment can be as follows: 1$)$ The resonant wavelengths corresponding to WGM resonances $\lambda_{i}^{\exp }(\mathrm{i}=1,2 \ldots, \mathrm{N})$ are determined from a PL spectrum of single microtube; 2) We assume an approximate value of the microtube radius based on the crosssectional SEM image; 3) Theoretical resonance positions $\lambda_{i}^{\text {theor }}$ are then calculated using eqs. (3) and (4); 4) The two lists are compared and for each value of $\lambda_{i}^{\text {exp }}$, the closest value $\lambda_{i}^{\text {theor }}$ is determined and the difference between them $\Delta_{\mathrm{i}}$ is calculated and 5) Taking into account the spectral resolution $\Delta$, the correlation $S=\frac{1}{N} \sum_{1}^{N}\left(1+\Delta_{i} / \Delta\right)^{-1}$ is then maximized by adjusting only two fitting parameters, namely the size of the microcylinder and the refractive index.

The results of the mode identification $\left(T M_{n}^{l}\right)$ for refractive index $=1.48$ and $\mathrm{D}=7.65 \mu \mathrm{m}$ are shown in Figure 5 . Agreement with the experimental values is excellent.

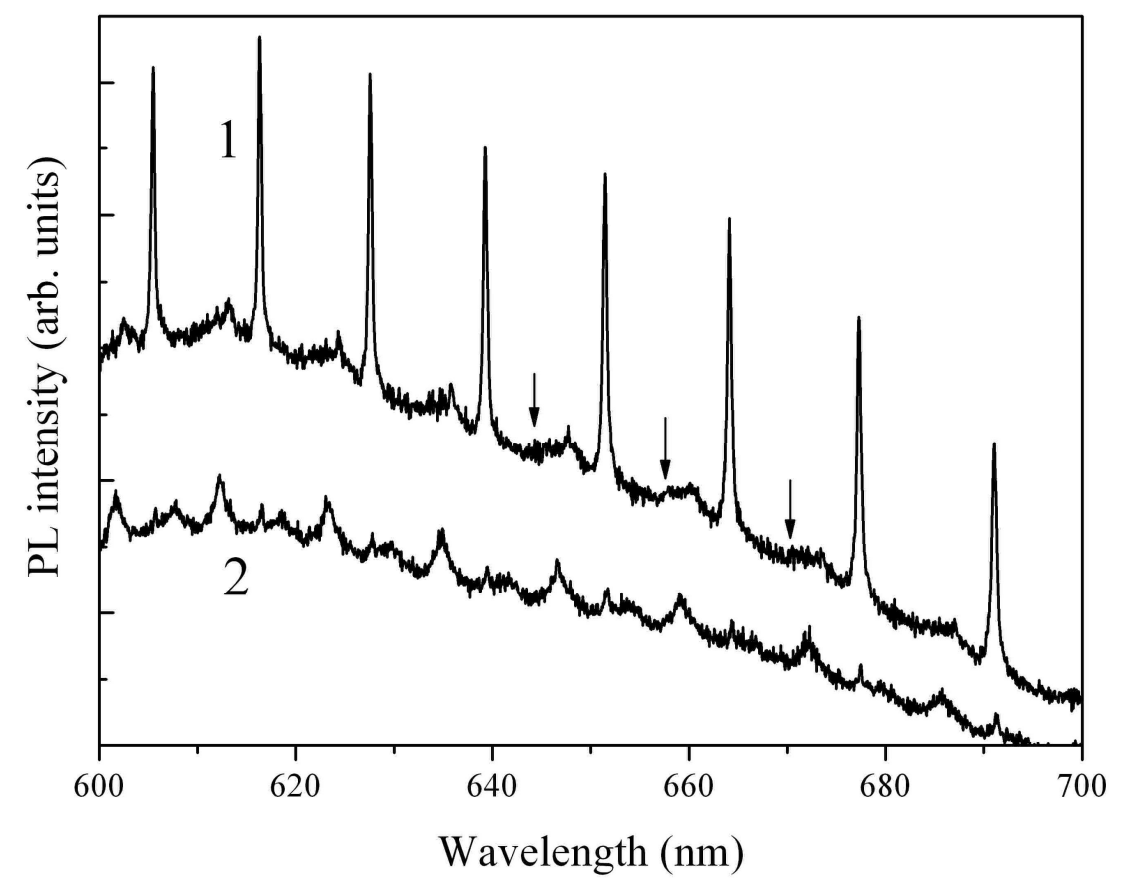

Figure 6. PL spectra of single free-standing microtube recorded (1) with polarizer orientation parallel to the microtube axis and (2).with polarizer rotated by $90^{\circ}$ 
The procedure described above for WGM identification allowed us to reveal another striking feature of the spectra of a single microtube - strong polarization properties. Experimentally, the distinction between modes of TE and TM polarization can be determined utilizing a polarizer inserted into the optical beam path in front of the detection system, which selects only the component of the electro-magnetic field parallel to the orientation of the polarizer. The sharp dominating peaks in the spectrum for polarizer orientation parallel to the microtube axis (Figure 6, curve 1) correspond to linear polarized light with the electric vector vibrating parallel to the axis of microtube. Rotating the polarizer by $90^{\circ}$ results in strong quenching of these WGMs (Figure 6, curve 2) unambiguously indicating their TM character.

A close look at the spectra presented in Figures 5 and 6 reveals the presence of satellites, broader peaks of the same polarization, which are blue-shifted with respect to the identified WGMs (indicated by arrows in Figure 6). The separation between these satellites homogeneously increases with wavelength ranging between 8 and $18 \mathrm{~nm}$ and is identical to the WGM spacings. Therefore, the observed secondary structure cannot be attributed to the WGM of higher $l$, for which smaller mode spacings are expected. Observation of two resonances of the same mode type can be explained by taking into account the fact that modes other than WGM can be supported by the microtube cavity. The presence of the second, inner surface in the microtube has a significant influence on the emission pattern of such microcavity systems. As a result, the microtube mode structure is more complex than in a single boundary microcavity such as a disc. Along with WGM originating from total internal reflection at the outer boundary there will be a different kind of mode penetrating the inner region at specific values of inner and outer diameter and refractive index. ${ }^{4}$ Weaker photon confinement will led to enhanced losses and therefore to a broadening of these modes. However, because WGMs are superimposed on a background PL signal (Figure 5), estimated value of photon storage time is too small to affect data of lifetime imaging. In fact as it shown above in Figure 4, the lifetime distribution detected from the side surface of microtube (Figure 4c) was very close to that measured from microtube tip (Figure 4d).

If we fit the WGM peaks by a Lorentzian function, we find quality factors defined by $\mathrm{Q}=\lambda_{0} / \Delta \lambda$ ranging between 2000 and 3200 with the maximum Q-value obtained for the peak centered at $616 \mathrm{~nm}$. The quality factor reflects how long a photon can be stored in the microcavity before leaking out. Since the cavity $Q$ factor is directly related to the lifetime of the photon in the cavity $\tau_{\text {cav }}=\mathrm{Q} / \omega_{0}$ (where $\omega_{0}$ is the resonant frequency), we can estimate a time constant $\tau_{\text {cav }} \approx 1 \mathrm{ps}$. It is noteworthy that $\mathrm{Q}$ values obtained at room temperature for the investigated microtube resonators are about the same magnitude as those recently reported for low-temperature $(T=5 K)$ spectra of semiconductor microtube ring resonators fabricated by optical lithography and wet-etching processes. ${ }^{22}$ This is the highest Q-factor achieved to date in the spectra of micro-cylinders or micro-tubes with diameter comparable to the present samples.

It is well known that the resonant internal field of a microcavity is not completely confined to the interior of the microresonator. Depending on the shape and size of the microcavity, the evanescent field can extend into the surroundings up to a couple of microns. It was recently recognized that the partial delocalization of Mie resonance states is of great importance, because it suggests the possibility for coherent coupling between WGMs of two adjacent microcavities with closely matched sizes. ${ }^{33,34}$ For both TE and TM polarizations, it was theoretically shown that the contribution of a resonant mode to the field outside the microcylinder decays exponentially with steeper decay predicted for the lower value of mode order $l .{ }^{35}$ In this present work we experimentally examine the distribution of this evanescent field. In Figure 7a, the integrated PL efficiency of a single microtube cavity is shown versus the excitation beam position scanning in the direction perpendicular to the microtube axis. It is clearly seen than the localization of the focal spot at the rim of the microtube (as compared to the central excitation) leads to an increase in integrated PL intensity mostly due to the glancing angle-of-incidence excitation with respect to the cavity surface. The results presented in Figure 7 clearly demonstrate that the strength and efficiency of the WGM are highly dependent on the excitation location. The evanescent field associated with cavity modes extends into the surroundings up to a distance comparable to the external radius of the microtube. This observation suggests a possibility to achieve strong evanescent coupling between adjacent high- $Q$ microresonators, and therefore, implies manifold applications, in biosensing, optical buffering and quantum information processing. 

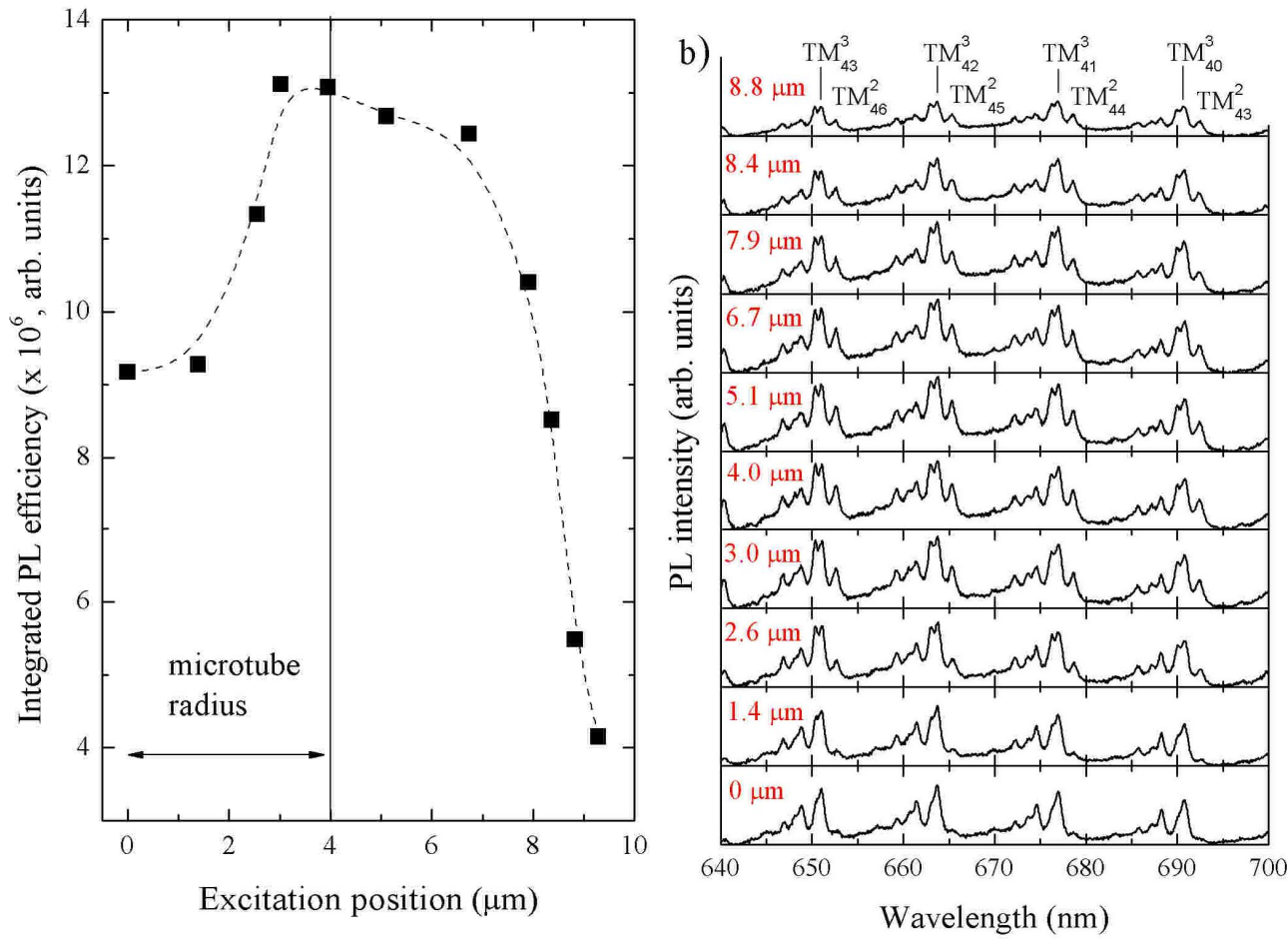

Figure 7. (a) Total luminescence intensity versus excitation beam position in single microtube. Position $0 \mu \mathrm{m}$ corresponds to the excitation point located on the axis of microtube. (b) Emission spectra for different locations of excitation spot. Vertical scale is the same for all panels. Background PL has been subtracted in all spectra presented.

\section{CONCLUSIONS}

We have developed a simple and robust technique for fabricating luminescent aluminosilicate microtubes and we have demonstrated the high-Q resonance modes in the emission spectra of a single microcavity. The method of preparation of such structures has unique advantages providing high-quality small three-dimensional microcavities with strongly polarized emission. The large evanescent field associated with cavity modes was found to extend into the surroundings up to a distance comparable to the external radius of the microtube. Potential applications for these alumosilicate microresonators will be in the areas of microlasers and microsensors and quantum information processing.

Acknowlegements: We thank the State Optical Institute, St. Petersburg for the supply of the micro-channel glass samples. The work was carried out with financial support of Science Foundation Ireland under the CRANN Nanotechnology Centre project PR04 and the Frontiers Research Programme.

\section{REFERENCES}

1. $\quad$ W. von Klitzing, R. Long, V. S. Ilchenko, J. Hare, V. Lefèvre-Seguin, New. J. Phys. 2001, 3, 14 .1.

2. $\quad$ K. J. Vahala, Nature 2003, 424, 839

3. $\quad$ H.-J. Moon, Y.-T. Chough, K. An, Phys. Rev. Lett. 2000, 85, 3161.

4. H.-J. Moon, G.-W. Park, S.-B. Lee, K. An, J. H. Lee, Opt. Commun. 2004, 235, 401. 
5. S. Arnold, Am. Sci. 2001, 89, 414.

6. A. B. Matsko, V. S. Ilchenko, IEEE J. Sel. Top. Quantum Electron 2006, 12, 3.

7. V. S. Ilchenko, A. B. Matsko, IEEE J. Sel. Top. Quantum Electron 2006, 12, 15.

8. Y. P. Rakovich, J. F. Donegan, N. Gaponik, A. L. Rogach, Appl. Phys. Lett. 2003, 83, 2539.

9. R. A. Wallingford, A. G. Ewing, Anal. Chem. 1988, 60, 1972-1975.

10. S. Blair, Y. Chen, Appl. Opt. 2001, 40, 570.

11. K. Mitsuhashi, N. Tagami, K. Tanabe, T. Ohkubo, H. Sakai, M. Koishi, M. Abe, Langmuir $2005,21,3659$.

12. R. M. Penner, C. R. Martin, Anal. Chem. 1987, 59, 2625.

13. J. Choi, G. Sauer, K. Nielsch, R. B. Wehrspohn, U. Gosele, Chem. Mater. 2003, 15, 776.

14. C. D. Merritt, B. L. Justus, Chem. Mater. 2003, 15, 2520.

15. S. A. Sapp, D. T. Mitchell, C. R. Martin, Chem. Mater. 1999, 11, 1183.

16. Y. L. Tai, H. Teng, Chem. Mater. 2004, 16, 338.

17. M. Wirtz, C. R. Martin, Adv. Mater. 2003, 15, 455.

18. D. J. Monk, D. R. Walt, J. Am. Chem. Soc. 2004, 126, 11416.

19. F. Namavar, F. Lu, C. H. Perry, A. Cremins, N. M. Kalkhoran, R. A. Soref, J.Appl. Phys. 1995, 77, 4813.

20. T. Kimura, A. Yokoi, H. Horiguchi, R. Saito, T. Ikoma, A. Sato, Appl. Phys.Lett. 1994, 65, 983.

21. A. M. Dorofeev, N. V. Gaponenko, V. P. Bondarenko, E. E. Bachilo, N. M.Kazuchits, A. A. Leshok, G. N. Troyanova, N. N. Vorosov, V. E. Borisenko, H. Gnaser, W. Bock, P. Becker, H. Oechsner, J. Appl. Phys. 1995, 77, 2679.

22. T. Kipp, H. Welsch, C. Strelow, C. Heyn, D. Heitmann, Phys. Rev. Lett. 2006, 96, 077403.

23. M. Steinhart, J. H. Wendorff, A. Greiner, R. B. Wehrspohn, K. Nielsch, J. Schilling, J. Choi, U. Gosele, Science 2002, 296, 1997.

24. W. H. Green, K. P. Le, J. Grey, T. T. Au, M. J. Sailor, Science 1997, 276, 1826.

25. M. R. Eftink, in Topics in Fluorescence Spectroscopy, Vol. 2 (Ed: J. R. Lakowicz), Plenum Press, New York 1991.

26. J. N. Demas, B. A. DeGraff, Sensors and Actuators B 1993, 11, 35.

27. D. R. James, Y.-S. Liu, P. De Mayo, W. R. Ware, Chem. Phys. Lett. 1985, 124, 460.

28. E. R. Carraway, J. N. Demas, B. A. DeGraff, Anal. Chem. 1991, 63, 332.

29. T. Förster, Disc. Faraday Soc. 1959, 27, 7.

30. H. C. Van de Hulst, Light Scattering by Small Particles, John Wiley \& Sons, New York 1957.

31. M. Kerker, E. Matijevic, J. Opt. Soc. Am. 1961, 51, 506.

32. P. W. Barber, S. C. Hill, Light scattering by particles: computational methods, World Scientific, Singapore 1990.

33. S. Deng, W. Cai, V. Astratov, Opt. Express 2004, 12, 6468.

34. Y. P. Rakovich, J. F. Donegan, M. Gerlach, A. L. Bradley, T. M. Connolly, J.J. Boland, N. Gaponik, A. Rogach, Phys. Rev. A 2004, 70, 051801(R).

35. J. F. Owen, R. K. Chang, P. W. Barber, Opt. Lett. 1981, 6, 540. 